\title{
Portal Vein Embolization: Tailoring, Optimizing, and Quantifying an Invaluable Procedure in Hepatic Surgery
}

\author{
Jean-Nicolas Vauthey, MD and Takashi Mizuno, MD, PhD \\ Department of Surgical Oncology, The University of Texas MD Anderson Cancer Center, Houston, TX
}

In this issue, Yamashita et al. ${ }^{1}$ present the experience of the University of Tokyo with portal vein embolization (PVE) in 338 patients from 1995 to 2013. The authors should be congratulated for presenting an extensive review of their indication, technique, and outcome of PVE. Over the past two decades, PVE has become an invaluable procedure in hepatic surgery. This editorial is an opportunity to revisit the indications for the procedure, the techniques to optimize the hypertrophy, and the methods to quantify its effects on regeneration.

In the study, Yamashita et al. used as indication for PVE a cut-off future liver remnant (FLR) volume of $<40 \%$ in patients with normal liver function based on an indocyanine green retention rate at $15 \mathrm{~min}$ (ICGR15) of $<10 \%$. This cautious approach was associated with an excellent outcome following resection, with an hepatic insufficiency rate of $2 \%$ and 90 -day mortality of $0.8 \%$. Anatomically, the left liver, on average, accounts for $33 \%$ of the total liver volume (TLV), and a $40 \%$ cut-off implies that the majority of patients undergoing right hepatectomy should receive PVE, which seems unnecessary. ${ }^{2}$ As such, the $40 \%$ cut-off value for PVE is higher than the 20-30\% used in the West, and the study may include more PVEs than clinically indicated. In our own experience with 301 extended right hepatectomies in patients with normal liver function, we demonstrated that patients with $20-30 \%$ FLR had similar postoperative outcomes compared with patients with $30-40 \%$ FLR, and only the subset of patients with $\leq 20 \%$ FLR had increased hepatic insufficiency and perioperative mortality. ${ }^{3}$ At MD Anderson Cancer Center, we

(C) Society of Surgical Oncology 2017

First Received: 4 January 2017;

Published Online: 7 February 2017

J.-N. Vauthey, MD

e-mail: jvauthey@mdanderson.org recommend PVE for FLR of $\leq 20 \%$ in normal liver, $\leq 30 \%$ in injured liver, and $\leq 40 \%$ in fibrosis/cirrhosis. ${ }^{4,5}$ Using appropriate indications for PVE, tailoring the procedure based on accurate volumetry and avoiding overutilization are important because PVE remains a procedure with a $7.8 \%$ risk of associated complications, as reported in the series of the University of Tokyo.

Yamashita et al. report an excellent degree of hypertrophy (median 10\%) and kinetic growth rate (3.9-4.5\% per week). In contrast, they report a median regeneration rate of $25 \%$ following PVE, which was lower than the regeneration rate of $62 \%$ following PVE in a recent series of 103 patients undergoing resection of colorectal liver metastases with small liver remnants. ${ }^{6}$ The lower regeneration rate in the series from the University of Tokyo may be attributed to generous indications for PVE and the performance of PVE in patients with an already large FLR unlikely to undergo significant regeneration. The technique recently used at the University of Tokyo is alcohol injection, which has led to less recanalization of the portal vein. It would have been interesting for the authors to compare the hypertrophy rates between alcohol injection and Gelfoam plus coil particle previously used by the authors. An important aspect of the technique at the University of Tokyo is the minimal use of segment 4 embolization (6/ $319,1.9 \%$ ) in spite of a large number of extended right hepatectomies $(116 / 256,45.3 \%)$. A major issue of right PVE without segment 4 embolization prior to extended right hepatectomy is the undesirable segment 4 regeneration at the expense of lesser regeneration of the left lateral bisegment. To counter this undesirable effect, at Nagoya University and MD Anderson Cancer Center, right PVE with segment 4 embolization has been used with a significant increase in the hypertrophy of the left lateral bisegment, compared with right PVE without segment 4 embolization. ${ }^{7,8}$ 
Yamashita et al. should be congratulated for presenting a detailed reporting of the volumetric outcome following PVE. The initial liver measurement includes the FLR before and after PVE and the TLV based on computed tomography. The FLR is expressed as a percentage of TLV. Alternatively, TLV can be derived from a formula based on body surface area, and the FLR is reported as the standardized FLR (sFLR). ${ }^{9}$ This latter method of measurement avoids the mistakes associated with subtraction of multiple tumors, the inclusion of nonfunctional liver with dilated ducts in the measurements, and the use of a different denominator between pre- and post-PVE total liver volume. The second measurement is the degree of hypertrophy-the difference between the pre- and postPVE measurement. This difference should be $\geq 5 \%$ to ensure a postoperative outcome with minimal postoperative hepatic dysfunction. ${ }^{10}$ The third relevant measurement is the kinetic growth rate-the degree of hypertrophy divided by time (weeks) elapsed between the pre- and postPVE measurements. Shindoh et al. reported that a kinetic growth rate $\geq 2 \%$ per week was associated with no liver failure-related deaths in a series of 103 patients with colorectal liver metastases. ${ }^{11}$ The kinetic growth rate has become an invaluable functional tool to assess the FLR. A kinetic growth rate $\geq 2 \%$ per week predicts a favorable outcome even in patients sustaining complications, and appears to protect patients with infectious complications from progressing from hepatic dysfunction to liver failure.

Similar to other reports, Yamashita et al. indicated that $20 \%$ (63/319) of patients receiving PVE did not ultimately undergo resection, which was due to progression of disease in the majority of patients (76\%) rather than the lack of regeneration (3\%). This is a reflection of the patient population undergoing PVE. Patients typically have advanced cancer, often compounded by unfavorable tumor biology, and progress in the interval between PVE and resection. ${ }^{12}$ Most patients undergoing PVE have adequate FLR hypertrophy, and only a minority do not have adequate liver regeneration after PVE. In such patients, we do not recommend Associating Liver Partition and Portal vein Ligation for Staged hepatectomy (ALPPS) because of its high morbidity rate. Alternative approaches in patients with inadequate hypertrophy after PVE are (i) preservation of segment 4 as part of the FLR and subsequent ablation of the liver metastases following right hepatectomy as completion ablation; ${ }^{13}$ (ii) consideration of additional embolization of the right or middle hepatic vein $;{ }^{14}$ or (iii) liver ablation using proton therapy. ${ }^{15}$

In conclusion, Yamashita et al. are commended for the third largest single-institutional series of PVE in the literature. ${ }^{16,17}$ PVE has increased the number of patients who are candidates for major hepatectomy. In addition, PVE measurement can be used as a functional test to predict hepatic dysfunction, hepatic insufficiency, and liver-related liver failure. In spite of early fears regarding the risks of cancer growth associated with the release of hepatotrophic factors, the long-term outcome of patients undergoing PVE after resection of colorectal liver metastases or hepatocellular carcinoma is similar to the long-term outcome of matched patients not undergoing PVE prior to resection. ${ }^{18,19}$

ACKNOWLEDGMENT Funding was provided by National Cancer Institute (Grant No. CA016672).

\section{REFERENCES}

1. Yamashita S, Yoshihiro S, Yamamoto S, et al. Efficacy of preoperative portal vein embolization among patients with hepatocellular carcinoma, biliary tract cancer, and colorectal liver metastases: a comparative study based on single center experience of 319 cases. Ann Surg Oncol. 2017. In press.

2. Abdalla EK, Denys A, Chevalier P, et al. Total and segmental liver volume variations: implications for liver surgery. Surgery. 2004;135:404-10.

3. Kishi Y, Abdalla EK, Chun YS, et al. Three hundred and one consecutive extended right hepatectomies: evaluation of outcome based on systematic liver volumetry. Ann Surg. 2009;250: 540-48.

4. Zorzi D, Laurent A, Pawlik TM, et al. Chemotherapy-associated hepatotoxicity and surgery for colorectal liver metastases. $\mathrm{Br} \mathrm{J}$ Surg. 2007;94:274-86.

5. Shindoh J, Tzeng CW, Aloia TA, et al. Optimal future liver remnant in patients treated with extensive preoperative chemotherapy for colorectal liver metastases. Ann Surg Oncol. 2013;20:2493-500.

6. Shindoh J, Vauthey JN, Zimmitti G, et al. Analysis of the efficacy of portal vein embolization for patients with extensive liver malignancy and very low future liver remnant volume, including a comparison with the associating liver partition with portal vein ligation for staged hepatectomy approach. J Am Coll Surg. 2013;217:126-33.

7. Nagino M, Kamiya J, Kanai M, et al. Right trisegment portal vein embolization for biliary tract carcinoma: technique and clinical utility. Surgery. 2000;127:155-60.

8. Kishi Y, Madoff DC, Abdalla EK, et al. Is embolization of segment 4 portal veins before extended right hepatectomy justified? Surgery. 2008;144:744-51.

9. Vauthey JN, Abdalla EK, Doherty DA, et al. Body surface area and body weight predict total liver volume in Western adults. Liver Transpl. 2002;8:233-40.

10. Ribero D, Abdalla EK, Madoff DC, et al. Portal vein embolization before major hepatectomy and its effects on regeneration, resectability and outcome. Br J Surg. 2007;94:1386-94.

11. Shindoh J, Truty MJ, Aloia TA, et al. Kinetic growth rate after portal vein embolization predicts posthepatectomy outcomes: toward zero liver-related mortality in patients with colorectal liver metastases and small future liver remnant. J Am Coll Surg. 2013;216:201-09.

12. Passot G, Chun YS, Kopetz SE, et al. Predictors of safety and efficacy of 2-stage hepatectomy for bilateral colorectal liver metastases. J Am Coll Surg. 2016;223:99-108.

13. Odisio BC, Yamashita S, Frota L, et al. Planned treatment of advanced metastatic disease with completion ablation after hepatic resection. J Gastrointest Surg. Epub 23 Nov 2016.

14. Hwang S, Lee SG, Ko GY, et al. Sequential preoperative ipsilateral hepatic vein embolization after portal vein embolization to 
induce further liver regeneration in patients with hepatobiliary malignancy. Ann Surg. 2009;249:608-16.

15. Colbert LE, Cloyd JM, Koay E, et al. Proton beam radiation as salvage therapy for bilateral colorectal liver metastases not amendable to second-stage hepatectomy. Surgery. Epub 9 Jan 2017.

16. Yokoyama Y, Ebata T, Igami T, et al. The adverse effects of preoperative cholangitis on the outcome of portal vein embolization and subsequent major hepatectomies. Surgery. 2014;156:1190-6.

17. Shindoh J, Tzeng CW, Aloia TA, et al. Safety and efficacy of portal vein embolization before planned major or extended hepatectomy: an institutional experience of 358 patients. $J$ Gastrointest Surg. 2014;18:45-51.

18. Shindoh J, Tzeng CW, Aloia TA, et al. Portal vein embolization improves rate of resection of extensive colorectal liver metastases without worsening survival. Br J Surg. 2013;100:1777-83.

19. Palavecino M, Chun YS, Madoff DC, et al. Major hepatic resection for hepatocellular carcinoma with or without portal vein embolization: perioperative outcome and survival. Surgery. 2009;145:399-405. 